\title{
Questes
}

vestes Revue pluridisciplinaire d'études médiévales

$36 \mid 2017$

Faire de l'histoire au Moyen Âge

\section{Faire de l'histoire : introduction}

Pauline Bouchaud, Mélanie Fougre-Lévêque et François Wallerich

\section{Q OpenEdition}

\section{Journals}

\section{Édition électronique}

URL : http://journals.openedition.org/questes/4431

DOI : 10.4000/questes.4431

ISSN : 2109-9472

Éditeur

Les Amis de Questes

Édition imprimée

Date de publication : 20 juin 2017

Pagination : 131-136

ISSN : 2102-7188

\section{Référence électronique}

Pauline Bouchaud, Mélanie Fougre-Lévêque et François Wallerich, «Faire de l'histoire : introduction », Questes [En ligne], 36 | 2017, mis en ligne le 02 juillet 2017, consulté le 24 mars 2020. URL : http:// journals.openedition.org/questes/4431 ; DOI : https://doi.org/10.4000/questes.4431 


\section{Introduction*}

\section{Pauline BouCHAUd, Mélanie Fougre-LEVEQUE et François WALLERICH}

École pratique des hautes études, Université Paris-Sorbonne et Université Paris-Nanterre

Depuis l'ouvrage pionnier de Bernard Guenée, Histoire et culture historique dans l'Occident médiéval ${ }^{1}$, les travaux sur l'historiographie médiévale se sont multipliés ${ }^{2}$ et ont permis de réhabiliter le travail des historiens de cette période qui n'étaient pas les chroniqueurs naïfs qu'on a longtemps dépeints.

Au Moyen Âge, l'histoire n'est ni une science, ni une discipline à part entière. Elle ne figure pas parmi les sept arts libéraux étudiés à l'université et n'est enseignée qu'en tant que discipline auxiliaire, dépendante de la grammaire, de la rhétorique ou encore de la théologie. Aucun auteur médiéval n'a songé à la théoriser dans un traité

\footnotetext{
* Nous adressons tous nos remerciements à Monsieur le Professeur Franck Collard qui a accepté de préfacer ce bulletin. Nous tenons également à remercier nos intervenants, les responsables de la revue ainsi que tous les Questeurs qui ont enrichi la réflexion autour de ce thème lors des discussions qui ont suivi les différentes interventions.

${ }^{1}$ Bernard Guenée, Histoire et culture historique dans l'Occident médiéval, Paris, Aubier, 1980.

${ }^{2}$ Citons, parmi les derniers parus : Bernard Guenée, Comment on écrit l'histoire au XIII ${ }^{e}$ siècle. Primat et le Roman des Roys, éd. Jean-Marie Moeglin, Paris, Éditions du CNRS, 2016; L'Écriture de l'histoire au Moyen Âge. Contraintes génériques, contraintes documentaires, dir. Étienne Anheim, Pierre Chastang, Francine MoraLebrun et Anne Rochebouet, Paris, Classiques Garnier, 2015 ; Pierre Courroux, L'Écriture de l'histoire dans les chroniques françaises (XII ${ }^{e}-X V^{e}$ siècle), Paris, Classiques Garnier, 2016.
} 
épistémologique $^{3}$. Néanmoins, l'histoire a ses règles propres qui s'inscrivent dans la pratique : elles prennent leurs sources dans les traités rédigés par les grands penseurs de l'Antiquité, Cicéron notamment ${ }^{4}$, et sont rappelées, sous la forme de poncifs, dans les préfaces des œuvres historiques médiévales ${ }^{5}$. Quelles sont-elles ? L'historien doit suivre l'ordre chronologique, décrire les lieux qu'il évoque et surtout dire la vérité quoiqu'il lui en coûte. L'histoire est avant tout un récit du vrai ${ }^{6}$, ou plutôt une recherche de la vérité car la connaissance pleine et entière du passé appartient à Dieu seul et l'historien ne peut que chercher à reconstruire ce passé de la manière la plus exacte possible. L'histoire a ses règles, elle a aussi son utilité, comme ne manquent pas de le souligner les historiens médiévaux, reprenant à l'envi la formule cicéronienne «Historia vero testis temporum, lux veritatis, vita memoriae, magistra vitae, nuntia vetustatis ${ }^{7} \gg$. Si l'histoire est parfois assimilée à un divertissement, elle a avant tout une fonction didactique et morale : elle doit conserver la mémoire des faits dignes d'être rappelés et fournir des exemples édifiants, des conseils de vie à ses lecteurs.

Après avoir brièvement évoqué le statut, les règles et les fonctions de l'histoire au Moyen Âge, intéressons-nous à ceux qui la pratiquent.

\footnotetext{
${ }^{3}$ Voir Pierre Courroux, L'Écriture de l'histoire dans les chroniques françaises (XII $-X V^{e}$ siècle), op. cit., p. 61.

${ }^{4}$ Voir Cicéron, De Oratore, éd. et trad. Edmond Courbaud, Paris, Les Belles Lettres, 1927, II, 62-63.

${ }^{5}$ Voir, au sujet des prologues, Les Prologues médiévaux, Actes du Colloque international organisé par l'Academia Belgica et l'École française de Rome avec le concours de la F.I.D.E.M. (Rome, 26-28 mars 1998), éd. Jacqueline Hamesse, Turnhout, Brepols, 2000.

${ }^{6}$ Isidore de Séville le rappelle dans ses Étymologies: «Historia est narratio rei gestae », «L'histoire est le récit de ce qui s'est passé » (Isidore de Séville, Étymologies, I, 41, cité d'après Bernard Guenée, Histoire et culture historique dans l'Occident médiéval, Paris, Aubier, 2011 [1980], p. 18).

${ }^{7}$ Cicéron, De Oratore, op. cit., II, 36 : «L'histoire enfin, témoin des temps, lumière de la vérité, vie de la mémoire, maîtresse de vie, messagère du passé ».
} 


\section{Diversité des profils d'historiens}

Au Moyen Âge, nombreux sont ceux qui font de l'histoire mais rares sont les hommes qui se consacrent à cette activité de manière exclusive ou professionnelle ${ }^{8}$. Pour cette raison, les historiens médiévaux ont des profils extrêmement divers comme le soulignent les nombreuses études monographiques parues depuis les années $1990^{9}$. Il est donc nécessaire, à qui veut étudier l'écriture de l'histoire, de prêter une attention particulière aux individus qui la font et de s'attacher à comprendre leur conception de l'histoire et la manière dont ils écrivent celle-ci.

L'Antiquité a légué à la période médiévale deux modèles d'historiens. Le premier est celui de l'historien-témoin : il renvoie à l'étymologie même du mot «histoire», dont le sens premier est «ce qu'on sait pour avoir été témoin ${ }^{10} »$. Ce modèle de l'historien qui a participé - ou du moins assisté - aux événements qu'il relate est par exemple celui du chevalier qui, à la fin de sa vie, troque l'épée contre la

\footnotetext{
${ }^{8}$ Bernard Guenée, Histoire et culture historique dans l'Occident médiéval, op. cit., p. 45. Ces derniers existent néanmoins : citons l'exemple des indiciaires de la cour de Bourgogne à la fin du Moyen Âge, qui sont rétribués pour écrire l'histoire de cette maison. À ce sujet, voir notamment Jean Devaux, Jean Molinet, indiciaire bourguignon, Paris, Champion, 1996.

${ }^{9}$ Parmi celles-ci, nous pouvons citer : Franck Collard, Un Historien au travail à la fin $d u X V^{e}$ siècle : Robert Gaguin, Genève, Droz, 1996; Isabelle Guyot-Bachy, Le Memoriale historiarum de Jean de Saint-Victor. Un historien et sa communauté au début du XIV siècle, Turnhout, Brepols, 2000 ; Anne-Marie Lamarrigue, Bernard Gui (1261-1331). Un historien et sa méthode, Paris, Honoré Champion, 2000 ; MarieMadeleine Dubrasquet-Pardo, Alfonso de Palencia, historien : études sur les Gesta hispaniensia, Villeneuve-d'Ascq, Presses universitaires du Septentrion, 2002. En outre, les dates anniversaires des historiens sont souvent l'occasion de colloques et de publications qui leur sont consacrés. Citons : Otto von Freising, Rahewin, Conradus sacrista. Geschichtsschreiber des 12. Jahrhunderts in Freising. Beiträge zum 850. Todesjahr Bischof Ottos von Freising 2008, dir. Ulrike Götz, Freising, Verlag des Historichen Vereins, 2010 ; Sigebert de Gembloux, dir. Jean-Paul Straus, Turnhout, Brepols, 2015.

${ }^{10}$ Historia est « formé sur le verbe historein, lui-même dérivé de histôr, le témoin, qui renvoie à idein, voir, et à (w)oida, savoir » : Pierre Courroux, op. cit., cit. p. 65 .
} 
plume pour narrer les batailles auxquelles lui et ses ancêtres ont jadis participé. Il est très vite concurrencé par le modèle de l'historien retiré du monde, qui a du recul sur les faits qu'il rapporte, incarné par les moines qui, depuis leur désert, décrivent les événements qui agitent le siècle. Ces deux modèles complémentaires perdurent durant tout le Moyen Âge.

À cette première distinction, il faut ajouter celles découlant du statut, de la fonction, des perspectives, de la culture historique de ceux qui font de l'histoire au Moyen Âge. Bernard Guenée propose ainsi de classer les historiens médiévaux en quatre grands groupes ${ }^{11}$. Le premier ensemble (tant du point de vue de la chronologie que de l'importance quantitative) regroupe ceux qui écrivent «à l'abri des cloîtres », c'est-àdire dans le cadre d'un établissement religieux, qu'il soit séculier ou régulier. À ce titre, si les évêques et les chapitres cathédraux se sont illustrés durant le haut Moyen Âge - notamment à travers le genre des gesta episcoporum ${ }^{12}$-, ils cèdent progressivement la place aux moines à partir de l'an mil ${ }^{13}$. Une telle évolution doit être reliée au dynamisme de la spiritualité monastique qui s'impose dans la vie religieuse de la société occidentale au XI ${ }^{\mathrm{e}}$ siècle $^{14}$. Les Bénédictins et, plus tard, les Cisterciens, composent, souvent de manière collective, une histoire érudite et savante dans ses méthodes mais une histoire qui est aussi avant tout « un sousproduit de la religion ${ }^{15} »$ puisque les citations de textes bibliques,

\footnotetext{
${ }^{11}$ Bernard Guenée, Histoire et culture historique dans l'Occident médiéval, op. cit., p. $44-76$.

${ }^{12}$ Signalons par exemple les gestes des évêques d'Auxerre: Les Gestes des évêques d'Auxerre, dir. Michel Sot, Paris, Les Belles lettres, 2002-2009, 3 tomes. Sur le genre des gesta episcoporum, voir en particulier Michel Sot, Gesta episcoporum, gesta abbatum, Turnhout, Brepols, 1981.

${ }^{13}$ L'historiographie épiscopale s'est davantage maintenue en terres d'Empire, avec les figures de Thietmar de Mersebourg ou encore d'Otton de Freising.

${ }^{14}$ Voir André Vauchez, La Spiritualité du Moyen Âge occidental. VIII ${ }^{e}$-XIII ${ }^{e}$ siècle, Paris, Seuil, 1994 [1975], p. 36.

${ }^{15}$ Bernard Guenée, «Y a-t-il une historiographie médiévale? », Revue historique, vol. 258, 1977, p. 261-275, cit. p. 266.
} 
patristiques, hagiographiques ou encore liturgiques y sont nombreuses. Fréquemment destinée à la communauté au sein de laquelle elle est produite, l'œuvre historique est l'occasion de faire l'éloge de l'établissement duquel elle émane ou encore de défendre les droits et possessions de celui-ci face aux prétentions d'un seigneur laïc ou ecclésiastique $^{16}$. À partir du XIII ${ }^{\mathrm{e}}$ siècle, les frères mendiants s'essaient au genre historique. Dans une optique de vulgarisation, éventuellement mise au service de la pastorale ${ }^{17}$, plusieurs d'entre eux, comme les dominicains Martin de Troppau ${ }^{18}$, Géraud de Frachet ${ }^{19}$ et Bernard Gui ${ }^{20}$ ou le franciscain Paolino da Venezia ${ }^{21}$, s'illustrent en composant des chroniques universelles ou encore, à l'instar de Vincent de Beauvais ${ }^{22}$, des ouvrages à vocation encyclopédique. Évêques, chanoines, moines et

${ }^{16}$ Pour un exemple d'historiographie monastique, voir la contribution de Thomas Ledru dans ce volume.

${ }^{17}$ L'aspect pastoral est particulièrement net chez les Franciscains, notamment chez Salimbene de Adam. Sur ce dernier, voir Olivier Guyotjeannin, Salimbene de Adam : un chroniqueur franciscain, Turnhout, Brepols, 1995.

${ }_{18}^{18}$ Martin de Troppau, Chronicon pontificum et imperatorum, éd. Ludwig Weiland, Monumenta Germaniae Historica (MGH), Scriptores, t. XXII, 1872, p. 397-475. Sur cette œuvre et son auteur, voir notamment Anna-Dorothee von den Brincken, «Martin von Troppau », dans Geschichtsschreibung und Geschichtsbewusstsein im späten Mittelalter, dir. Hans Patze, Sigmaringen, Thorbecke, 1987, p. 155-193.

${ }^{19}$ Voir Régis Rech, Géraud de Frachet : l'engagement d'un historien au XIII siècle. Édition de sa Chronique universelle, Thèse de diplôme d'archiviste-paléographe, Paris, École nationale des chartes, 1993.

${ }^{20}$ Pour une recension des manuscrits des Fleurs des chroniques de Bernard Gui (qui n'ont à ce jour pas été éditées), voir Léopold Delisle, « Notice sur les manuscrits de Bernard Gui », Notices et extraits des manuscrits de la Bibliothèque nationale et autres bibliothèques, t. XXVII, $2^{\mathrm{e}}$ partie, Paris, 1879, p. 169-455, et plus particulièrement p. 188-240.

${ }^{21}$ Pour une recension des manuscrits des trois chroniques universelles composées par Paolino da Venezia, à savoir l'Epithoma, le Compendium et la Satirica ystoria, qui sont demeurées inédites à l'exception de quelques extraits, voir Isabelle HeullantDonat, «Entrer dans l'Histoire. Paolino da Venezia et les prologues de ses chroniques universelles », Mélanges de l'École française de Rome, Moyen Âge - Temps modernes, t. $105, \mathrm{n}^{\circ} 1,1993$, p. 381-442, et plus particulièrement l'annexe $\mathrm{I}$, p. 426-435.

${ }^{22}$ Vincent de Beauvais, Speculum majus, Douai, 1624, 4 vol. Sur cette œuvre, voir en dernier lieu Monique Paulmier-Foucart et Marie-Christine Duchenne (collaboratrice), Vincent de Beauvais et le Grand Miroir du Monde, Turnhout, Brepols, 2004. 
frères mendiants ont en commun d'avoir choisi le latin comme langue de rédaction de leurs œuvres historiques.

Ils se distinguent en cela des historiens qui écrivent pour un patron dans le but d'en obtenir une rémunération, qui appartiennent au deuxième groupe établi par Bernard Guenée. En effet, les historiens de cour, qui fleurissent à partir du XII ${ }^{\mathrm{e}}$ siècle mais restent minoritaires tout au long de la période médiévale, adoptent vite la langue vulgaire pour plaire à leur public. Auteurs d'une « littérature de "propagande" ${ }^{, 23}$ », ils n'hésitent pas à reprendre les procédés littéraires de l'épopée pour chanter les louanges de leurs héros. Ils sont en effet avant tout des «gens de lettres », composant une histoire au caractère littéraire affirmé ${ }^{24}$.

Les $\mathrm{XIV}^{\mathrm{e}}$ et $\mathrm{XV}^{\mathrm{e}}$ siècles voient apparaître un type d'historien d'un genre nouveau, l'historien-bureaucrate. En effet, le développement des administrations au sein d'États de plus en plus centralisés entraîne l'essor de couches sociales nouvelles. La chancellerie et le notariat sont des exemples connus de milieux dans lesquels les laïcs sont de plus en plus nombreux à manier les techniques relatives à l'écriture ${ }^{25}$. Les historiens issus de ces milieux, qui ont pour habitude de manipuler les sources diplomatiques et ont pour passion l'érudition, s'appuient largement sur des documents d'archives qu'ils mettent au service d'un travail rigoureux

\footnotetext{
${ }^{23}$ Bernard Guenée, Histoire et culture historique dans l'Occident médiéval, op. cit., p. 64 .

${ }^{24}$ Pensons par exemple aux talents littéraires de Jean Lemaire de Belges, indiciaire bourguignon et historiographe de Marguerite d'Autriche à l'aube du XVI siècle, auteur des Illustrations de Gaule et singularités de Troie. Voir notamment à son sujet Pierre Jodogne, Jean Lemaire de Belges, écrivain franco-bourguignon, Bruxelles, Palais des Académies, 1972.

${ }^{25}$ Le notariat est particulièrement important dans les régions méditerranéennes. Voir la thèse récente de Matthieu Allingri, Le Métier de notaire en Europe méridionale à la fin du Moyen Âge. Étude comparée de deux modèles régionaux (Italie communale, pays catalans, v. 1280-1420), Thèse de doctorat, Lyon, Université Lumière Lyon 2, 2014.
} 
et méthodique ${ }^{26}$. Contrairement aux moines qui écrivent dans le but de faire leur salut et aux historiens de cour qui espèrent de leurs mécènes une rémunération, les historiens-bureaucrates n'attendent de leurs œuvres aucune contrepartie : « [leur] passion est gratuite ${ }^{27} »$.

Enfin, l'accès plus large à l'écrit et les progrès de la culture historique entraînent l'apparition d'un dernier type d'historien, l'amateur ${ }^{28}$. Travaillant avant tout par plaisir, celui-ci produit souvent une histoire locale dont il a été le témoin direct. Généralement moins érudite et moins livresque que l'histoire savante des notaires et autres chanceliers, reposant avant tout sur les sources orales et la mémoire familiale, et rédigée en langue vulgaire, son œuvre se rapproche souvent de l'autobiographie.

Si la typologie élaborée par Bernard Guenée permet de brosser à grands traits les évolutions de la pratique de l'histoire et la diversité sociologique des historiens, elle ne doit pas masquer la singularité de chaque individu : il est impossible de comprendre une œuvre historique sans tenir compte des choix personnels et de la culture historique de son auteur.

\section{Le travail de l'historien}

Chaque historien a sa propre conception de l'histoire, dont découle la méthode de travail qu'il adopte.

\footnotetext{
${ }^{26}$ Leonardo Bruni est un parfait représentant de cette histoire érudite. Chancelier de la ville de Florence et auteur d'une œuvre historique majeure, l'Historia Fiorentinarum libri XII, Bruni s'est également illustré par la traduction d'un nombre considérable d'œuvres de Platon en latin dans la première moitié $\mathrm{du} \mathrm{XV}$ siècle. Voir Gary Ianziti, Writing history in Renaissance Italy. Leonardo Bruni and the uses of the past, Cambridge/Londres, Harvard University Press, 2012.

${ }^{27}$ Bernard Guenée, Histoire et culture historique dans l'Occident médiéval, op. cit., p. 69.

${ }^{28}$ Voir l'exemple de Jean de Haynin présenté dans la contribution d'Anh Thy Nguyen dans ce volume.
} 


\section{La quête des sources}

L'historien, quel qu'il soit, doit tout d'abord rassembler la documentation nécessaire à l'écriture de son œuvre. Il peut avoir recours à différents types de sources dont la nature varie en fonction de la documentation dont il dispose, de sa culture historique, mais aussi de la période dont il traite dans son œuvre.

Pour faire l'histoire de l'époque qui lui est contemporaine, l'historien-témoin se sert avant tout de son expérience personnelle et de sa mémoire. Toutefois, celles-ci ne concernant qu'une partie infime des événements s'étant produits dans un passé proche, il doit également utiliser d'autres témoignages.

Le recours aux sources orales est une pratique largement partagée par les historiens médiévaux, quels qu'ils soient ${ }^{29}$. Ainsi n'hésitent-ils pas à s'appuyer sur le témoignage d'autrui - en prenant soin toutefois d'indiquer avoir interrogé des personnes « dignes de foi »- pour relater les événements qui se sont produits jusqu'à cinquante ans avant qu'ils ne prennent la plume. Pour les temps plus reculés (jusqu'à une centaine d'années avant la rédaction), ils peuvent utiliser la tradition orale. Ils ne négligent pas non plus la fama, la rumeur populaire, même s'ils la savent moins précise et davantage sujette à caution.

Tout au long du Moyen Âge, les historiens ont également recours aux livres qu'ils choisissent selon les perspectives qui sont les leurs : ils peuvent ainsi utiliser des chroniques, des histoires, des annales, des romans, des chansons de geste, des vies de saints, des récits de miracles, ou encore des textes liturgiques. Si certains d'entre eux se contentent des ouvrages que contient la bibliothèque de leur monastère, de leur église ou encore de la demeure de leur seigneur, d'autres n'hésitent pas à aller

\footnotetext{
${ }^{29}$ Sur l'importance des sources orales pour l'historien médiéval, voir Bernard Guenée, Histoire et culture historique dans l'Occident médiéval, op. cit., p. 78-85.
} 
consulter des manuscrits dans des dépôts extérieurs, comme Jean de Saint-Victor qui complète les informations qu'il a puisées dans le très riche fonds de son abbaye par des livres issues de bibliothèques d'autres églises, celle de Saint-Denis notamment ${ }^{30}$.

Nombreux sont aussi les historiens qui utilisent des sources diplomatiques $^{31}$. Les religieux le font dès le haut Moyen Âge, la pratique se répand ensuite hors des établissements ecclésiastiques et le document d'archives devient une source privilégiée aux XIV $-\mathrm{XV}^{\mathrm{e}}$ siècles $^{32}$.

Enfin, contrairement à ce que l'on a longtemps prétendu, les historiens du Moyen Âge se sont intéressés aux sources monumentales, qu'elles soient épigraphiques ou archéologiques : même s'ils n'ont pas encore les connaissances nécessaires pour déchiffrer correctement toutes les inscriptions ou dater les ruines et édifices qu'ils peuvent voir, certains, comme Aymeric de Peyrac ${ }^{33}$, ont un vrai goût des monuments dont témoignent les nombreuses descriptions qu'ils en ont laissées ${ }^{34}$.

\footnotetext{
${ }^{30}$ Isabelle Guyot-Bachy, op. cit., p. 164-168.

${ }^{31}$ C'est le cas du chanoine limousin Étienne Maleu qui achève son Liber chronicarum ecclesiae Sancti Juniani en 1316: outre de nombreuses analyses, il insère dans son œuvre la copie de vingt-sept chartes et bulles (Chronique de Maleu, chanoine de Saint-Junien mort en 1322, éd. François Arbellot, Saint-Junien/Paris, Barret/Victor Didron, 1847).

${ }^{32}$ Bernard Guenée, op. cit., p. 91-100.

33 Aymeric de Peyrac, Chronique des abbés de Moissac, éd. Régis de la Haye, Maastricht/Moissac, 1994. Voir Paul Mironneau, «Éloge de la curiosité. Aymeric de Peyrac (vers 1340-1406) », Cahiers de Fanjeaux, $\mathrm{n}^{\circ} 35$, Église et culture en France méridionale (XII ${ }^{e}-X I V^{e}$ siècle), 2000, p. 149-183.

${ }^{34}$ Il faut attendre la Renaissance pour que les historiens soient capables de tirer parti de ces monuments et inscriptions, grâce aux progrès de l'archéologie et de l'épigraphie (cf. Bernard Guenée, op. cit., p. 90). Les humanistes se passionnèrent ainsi pour l'observation des vestiges et leur identification à des fins de classification. Cette étude des ruines se retrouve par exemple dans les ouvrages du Pogge (on pense notamment à son opuscule sur Les ruines de Rome: Poggio Bracciolini, De Varietate Fortunae, Livre I. Les Ruines de Rome, éd. et trad. Jean-Yves Boriaud, Paris, Les Belles Lettres, 1999) ou dans la lettre de Pétrarque à son ami Giovanni Colonna qui célèbre en 1341 les lieux mémorables de la Rome antique (Francesco Petrarca, Le Familiari, éd. Vittorio Rossi, vol. II : Libri V-XI, Florence, G. C. Sansoni, 1934, VI, 2). Sur l'écriture de la ruine et le développement d'une proto-archéologie à la Renaissance, on consultera notamment les travaux de Clémence Revest comme «Se
} 


\section{Compilation et mise en récit}

Après que l'historien a rassemblé sa documentation, il doit opérer une sélection parmi ses sources, choisir lesquelles il va privilégier, quels faits il va rapporter, quelle version d'un même événement il va préférer, quels passages il va supprimer ou résumer, etc. Cette sélection répond à un critère de vérité : l'historien sélectionne les sources qu'il juge fiables, mais elle est également révélatrice de son projet, de sa conception de l'histoire ainsi que de la manière dont il reconstruit la vérité du passé.

Une fois ce tri opéré, l'historien doit construire son récit. L'organisation de la matière historique obéit certes à des contraintes génériques : l'auteur doit se plier aux lois du genre - annales, chronique, histoire, gesta - qu'il a choisi ${ }^{35}$, mais elle dépend également du projet de l'historien. Aucun récit historique n'est neutre. Tout choix, qu'il concerne le titre, le plan, le style, la langue de l'œuvre, est significatif et révélateur quant aux perspectives de l'historien, à sa conception de l'histoire et au public visé. L'historien écrit en effet avant tout pour un public et l'on sait que de grands auteurs, comme Jean Froissart, se sont souciés de la portée et de la diffusion de leurs œuvres ${ }^{36}$.

souvenir de Rome: humanisme et pédagogie de la mémoire dans l'Italie du $\mathrm{XV}^{\mathrm{e}}$ siècle ", Camenulae, 1, La mémoire, ses formes, ses structures (Journée de l'École doctorale 1 de l'Université Paris IV-Sorbonne, 12 mai 2007), novembre 2007, URL : http://www.paris-sorbonne.fr/IMG/pdf/C. Revest-2.pdf, page consultée le 20 mai 2017.

${ }^{35}$ Sur la question des différents genres historiques, voir Bernard Guenée, « Histoires, annales, chroniques. Essai sur les genres historiques au Moyen Âge », Annales. Économies, Sociétés, Civilisations, vol.4, 1973, p.997-1016 et Bernard Guenée, «Histoire et Chronique. Nouvelles réflexions sur les genres historiques au Moyen Âge », dans La Chronique et l'Histoire au Moyen Âge, Colloque des 24 et 25 mai 1982 organisé par le Département d'études médiévales de l'Université de ParisSorbonne, dir. Daniel Poirion, Paris, PUPS, 1984, p. 3-12.

${ }^{36}$ Sur les facteurs permettant de mesurer le succès et donc la portée d'une ouvre, voir Bernard Guenée, Histoire et culture historique dans l'Occident médiéval, op. cit., p. 248-299. Sur la question de la diffusion et de la réception des œuvres historiques au Moyen Âge, voir la contribution d'Antoine Brix dans ce volume. 
Le travail de compilation, autrefois dénigré, aujourd'hui réhabilité, nous renseigne lui aussi sur le projet de l'historien puisque ce dernier agence et combine, par le biais de diverses méthodes (simple juxtaposition de différents textes ou entrecroisement plus serré des sources, insertion dans le récit de brèves citations ou de longs extraits) les emprunts aux différentes sources en une construction savamment élaborée pour donner sa vision de la vérité du passé. Il peut manipuler les sources de manière consciente, voire recourir à son imagination et inventer, de manière vraisemblable, pour donner davantage de cohérence ou de sens à son récit (inventer des causes pour expliquer un phénomène incompris par exemple), le faire correspondre à sa vision du passé, combler les lacunes des sources ou atteindre le but d'édification qu'il s'est fixé vis-à-vis de ses lecteurs ${ }^{37}$.

Ainsi l'historien médiéval n'est-il ni naïf, ni crédule. L'organisation de son récit procède de choix réfléchis ; les modifications qu'il apporte à ses sources ainsi que les inventions auxquelles il se livre dans son œuvre sont conscientes et révélatrices de ses objectifs. Le récit fait sens: il nous dévoile la manière dont l'historien comprend et interprète le passé. Une œuvre historique médiévale est en effet «le produit d'une imagination subjective qui donne sa vision de l'histoire ${ }^{38} »$. En composant son récit, l'historien devient «le véritable maître du déroulement de l'histoire tel qu'il peut être connu», s'érige en juge du sens de l'histoire et construit donc la vérité historique en restituant «l'histoire telle qu'elle aurait dû être ${ }^{39} »$.

\footnotetext{
${ }^{37}$ Pierre Courroux évoque ainsi l'exemple de l'historien qui use d'anachronismes pour renforcer l'exemplarité des modèles qu'il propose : Pierre Courroux, L'Écriture de l'histoire dans les chroniques françaises (XII $-X V^{e}$ siècle), op. cit., p. 861-862.

${ }^{38}$ Ibid., cit. p. 856.

${ }^{39}$ Jean-Marie Moeglin, «La vérité de l'histoire et le moi du chroniqueur », dans La Vérité. Vérité et crédibilité : construire la vérité dans le système de communication de
} 


\section{Histoire et littérature : un lien problématique}

Par son caractère narratif et rhétorique et sa proximité avec des genres littéraires tels que la chanson de geste, le roman ${ }^{40}$ ou encore l'hagiographie, l'écriture de l'histoire entretient d'étroites relations avec la littérature ${ }^{41}$. Cette porosité suscite un certain nombre de questionnements. Faut-il, par exemple, distinguer historia et fabula ou admettre au contraire que l'une ne va pas sans l'autre ? La vérité est-elle nécessairement associée à l'histoire et le mensonge à la littérature ? La recherche de vérité propre à l'historiographie serait-elle incompatible avec la recherche d'un style inhérent à la littérature ? Ou la littérature conférerait-elle au contraire une légitimité plus grande à la matière historique?

L'histoire étant une pratique littéraire, faire de l'histoire au Moyen Âge, c'est d'abord écrire l'histoire, choisir un style. Dans la littérature latine médiévale, la séparation entre œuvre de fiction et œuvre historique est nette et l'historia, l'histoire, est traditionnellement opposée à la fabula, la fable, le mensonge. La fabula se caractérise par la recherche de l'élégance ; elle est traditionnellement associée à la poésie. L'histoire, qui se définit comme la recherche de la vérité, doit donner le récit littéral de ce qui s'est passé et, pour ce faire, employer les mots dans leur sens premier. Ainsi bon nombre de chroniqueurs de langue latine, monastiques en particulier, font-ils le choix d'un style simple qui permet,

l'Occident (XIII ${ }^{e}$-XVII ${ }^{e}$ siècle). Actes de la conférence organisée à Rome en 2012 par SAS en collaboration avec l'École française de Rome, dir. Jean-Philippe Genet, Paris/Rome, Publications de la Sorbonne/École française de Rome, 2015, p. 521-538, cit. p. 538.

${ }^{40}$ Voir la contribution de Justine Breton dans ce volume.

${ }^{41}$ Voir, par exemple, sur les liens entre histoire et théâtre, la contribution de Shanshan Lu dans ce volume. 
selon eux, de mieux dire la véritét ${ }^{42}$. Mais la tentation d'un style plus travaillé est grande et de nombreux auteurs y succombent tout en continuant de prétendre, dans leurs prologues ${ }^{43}$, avoir adopté une langue simple.

C'est dans la littérature française du XII siècle que l'opposition entre œuvre fictive et œuvre historique devient confuse: il existe désormais une « littérature historique ${ }^{44}$ » qui côtoie une histoire romancée.

Le problème des frontières génériques va d'abord de pair avec un problème de terminologie. Le terme médiéval estoire prend en effet des acceptions qui recouvrent aussi bien le domaine historique que littéraire puisqu'il peut signifier «source, histoire, récit» ou encore «récit en cours de composition» et peut même prendre le sens de « $\operatorname{roman}^{45} »$. À travers son caractère polysémique, l'estoire propose deux sortes de vérité (non exclusives l'une de l'autre), celle, référentielle, du chroniqueur, qui entretient un lien étroit avec le monde vécu, et celle du romancier, qui émane du sens de l'œuvre composée ${ }^{46}$.

Par ailleurs, le beau style n'est plus l'apanage des œuvres fictives. Les historiens de langue française sont soucieux de plaire à leur public ; ils font donc montre de leurs talents littéraires, répondant à leurs détracteurs que la beauté du style ne s'oppose pas à la véracité du récit

\footnotetext{
${ }^{42}$ Bernard Guenée, Histoire et culture historique dans l'Occident médiéval, op. cit., p. 215.

${ }^{43}$ Sur le caractère formel des prologues dans les chroniques et la stratégie discursive qui s'y dessine, on lira avec intérêt l'article de Christiane Marchello-Nizia, «L'historien et son prologue : forme littéraire et stratégies discursives », dans $L a$ Chronique et l'Histoire au Moyen Âge, op. cit., p. 13-25.

${ }^{44}$ Bernard Guenée, Histoire et culture historique dans l'Occident médiéval, op. cit., cit. p. 221.

45 Catherine Croisy-Naquet, «Approches historiennes, approches littéraires. L'exemple de l'Estoire de la guerre sainte », dans L'Écriture de l'histoire au Moyen Âge, op. cit., p. 21-36, cit. p. 21.

${ }^{46}$ Pierre Courroux établit une distinction entre «vérité référentielle » et «vérité de sens » dans l'article «"Si com jou truis el livre escrit". Les références externes dans la Chronique rimée de Philippe Mousket », dans L'Écriture de l'histoire au Moyen Âge, op. cit., p. 161-174, cit. p. 161.
} 
mais permet, au contraire, de mieux soutenir la vérité, en apportant notamment une densité nouvelle à la dimension informative et concrète de l'historiographie. Elle amplifie, augmente le langage technique de l'historien pour ajouter à l'exactitude des faits la force de l'émotion ${ }^{47}$. Cette actualisation des événements à travers l'émotion joue plusieurs rôles essentiels à la construction et à la réception du récit historique : elle a vocation à susciter l'intérêt du lecteur et à favoriser la dimension mémorielle de l'histoire. La poétique du récit n'amoindrit en rien la conscience historique de l'auteur mais offre un supplément de sens à l'historiographie en ce qu'elle permet au lecteur de s'approprier le texte. La rhétorique, les choix stylistiques et les modèles narratifs convoqués transcendent l'aspect documentaire en dotant le passé de la dimension vécue et, à l'occasion, en dévoilant l'orientation idéologique du texte.

Le style trahirait-il de ce fait la vérité de l'histoire ? Il semble que la notion même de vérité doive être redéfinie à l'aune des pratiques qui forgent l'historiographie. En effet, si l'historien travaille à montrer le vrai, l'influence des traditions existantes participe plus ou moins consciemment de cette construction du réel. Ce fonds littéraire raconte en parallèle une autre histoire, celle des mentalités et celle des croyances d'une communautét ${ }^{48}$. En somme, les corrélations entre l'historiographie et les récits de fiction, entre l'histoire et les histoires, donnent un surcroît de sens à une réalité historique rendue nécessairement subjective et équivoque par le regard de l'homme. La littérature, portée par l'intertextualité, apporte donc une dimension interprétative à l'écriture de

\footnotetext{
${ }^{47}$ Plusieurs auteurs rappellent ainsi que l'auctor est celui qui rehausse les faits par son style, qui en augmente (augere) la puissance: voir Bernard Guenée, Histoire et culture historique dans l'Occident médiéval, op. cit., p. 216.

${ }^{48}$ Catherine Croisy-Naquet, « Approches historiennes, approches littéraires », art. cit., p. 21-36, p. 34.
} 
l'histoire. Les mots témoignent du réel tout en le décryptant. Ils en disent la mouvance essentielle et, en cela, sont également parole de vérité.

L'histoire a ainsi vocation à conserver la mémoire des époques passées, à décrire ces époques à travers le récit de la succession des événements. Ce récit doit être simple et vrai. Écrire l'histoire n'est pas dire l'histoire. Si les poèmes de croisade peuvent constituer, pour la plupart, de précieux témoignages oculaires, renouant ainsi avec l'étymologie supposée du mot «historia », leurs dispositifs rhétorique et poétique empêchent d'en faire des œuvres d'historiographes. Ce serait en outre trahir l'intention du poète et oublier un peu vite qu'il existait déjà à l'époque une conscience générique dont les contours restaient, certes, encore à définir. Ces chansons de croisade participent pourtant de l'élaboration d'une conscience historique et remotivent par là-même le sens étymologique du mot «document» : «vecteur d'une leçon portant sur les faits, support d'une vision de l'histoire qui implique la distance critique $^{49} \gg$.

Ainsi, au-delà d'un sens littéral fidèle au déroulement des faits, le style adopté et les emprunts aux différents genres littéraires rendent perméable la frontière entre histoire et roman qui usent des mêmes procédés d'écriture alors que leurs objectifs divergent. Plus qu'une frontière, il faudrait en réalité y voir un continuum. L'histoire se dote alors d'un sens allégorique qui n'est pas sans rappeler les accointances de cette science avec une autre discipline, la théologie. Si la stratégie discursive diffère, histoire, littérature et théologie occupent néanmoins

\footnotetext{
${ }^{49}$ Florence Tanniou, «Mémoire des lettres et des lois. Modalités d'insertion et interprétation des traces documentaires dans les Mémoires de Philippe de Novare », dans L'Écriture de l'histoire au Moyen Âge, op. cit., p. 89-101, cit. p. 97. Document vient du latin docere: voir Francine Mora-Lebrun et Anne Rochebouet, «Introduction. In Praesentia: le genre historique au prisme du document », dans L'Écriture de l'histoire au Moyen Âge, op. cit., p. 7-20, p. 9.
} 
des fonctions similaires en ce qu'elles représentent des outils de pensée permettant d'appréhender le monde. Dans cette perspective, faire de l'histoire consisterait à conférer une intelligibilité aux événements en proposant, par des moyens divers, un « compte-rendu du réel ${ }^{50} »$.

${ }^{50}$ Vera Vějrychová, «La réécriture de l'histoire chez Jean Froissart. Le chroniqueur face à ses sources ", dans L'Écriture de l'histoire au Moyen Âge, op. cit., p. 103-114, cit. p. 103. 